\title{
226. Kombinationsbehandlung mit Cyclosporin A und Prednisolon bei klinischer Nierentransplantation
}

\author{
H. Bunzendahl, K. Wonigeit, E. Wagner und R. Pichlmayr \\ Klinik für Abdominal- und Transplantationschirurgie, Medizinische Hochschule Hannover, \\ Konstanty-Gutschow-Straße 8, D-3000 Hannover 61
}

\section{Cyclosporin A plus Prednisolone in Renal Transplantation}

\begin{abstract}
Summary. In 198275 renal transplant recipients ( 6 third transplants, 11 second transplants) were treated with cyclosporin A (CyA) and prednisolone. The CyA dose was initially $14 \mathrm{mg} / \mathrm{kg}$ post operative, reduced to a maintenance dose of $8 \mathrm{mg} / \mathrm{kg}$ after 2 weeks. Prednisolone was then added $(2.0 \mathrm{mg} / \mathrm{kg}$, fast reduction to $0.15 \mathrm{mg} / \mathrm{kg})$. After a follow-up of at least 4 months we found a dramatic reduction of rejection episodes $(22 \%$ of all patients). Graft survival (actual survival) at 12 months was $73 \%$ (no exclusions); side effects were few. We consider this protocol superior to other protocols previously used.
\end{abstract}

Key words: Cyclosporin A - Renal transplantation - Immunosuppression.

Zusammenfassung. 1982 wurden 75 erwachsene Nierentransplantatempfänger (6mal 3. Transpl., $11 \mathrm{mal}$ 2. Transpl.) mit einer Kombination aus Cyclosporin A (CyA) und Prednisolon behandelt. Die CyA Dosierung war $14 \mathrm{mg} / \mathrm{kg}$ p.o. in der ersten Woche postop., dann $11 \mathrm{mg} / \mathrm{kg}$ in der zweiten und $8 \mathrm{mg} / \mathrm{kg}$ als Dauertherapie. Prednisolon wurde hinzugefügt $(2,0 \mathrm{mg} / \mathrm{kg}$, schnelle Reduktion auf $0,15 \mathrm{mg} / \mathrm{kg})$. Bei einer Mindestbeobachtungszeit von 4 Monaten ergab sich eine drastische Reduktion der Abstoßungsepisoden auf insges. 22\% der Patienten. Die Transplantatfunktion nach 12 Mon. (act. surv.) was $73 \%$ (keine Ausschlüsse), die Nebenwirkungen gering. Wir halten diese Immunsuppression für die derzeit bestmögliche.

Schliisselwörter: Cyclosporin A - Nierentransplantation - Immunsuppression.

\section{Die spontane Nierentransplantatruptur und ihre operative Versorgung}

\author{
W. Niebel, H. D. Jakubowski, G. Dostal, F. W. Eigler
}

Abteilung für Allgemeine Chirurgie, Universitätsklinikum Essen, Hufelandstraße 55, D-4300 Essen

\section{Spontaneous Renal Allograft Rupture and Its Operative Treatment}

Summary. Among 501 renal transplantations (recipients $n=453$ ) a spontaneous rupture of the graft occurred in 25 patients. In 6 cases the graft had to be immediately removed because of irreversible rejection. In 19 patients the grafts could primarily be saved. In each case the effective surgical repair consisted of suturing the rupture with the use of muscle tissue. The insertion of a covering material over the rupture seems to be unnecessary. The long-term survival of repaired grafts has shown to be good.

Key words: Kidney transplantation - Renal allograft rupture.

Zusammenfassung. Bei 501 Nierentransplantationen (Empfänger $n=453$ ) fanden sich 25 Patienten mit einer spontanen Ruptur des Transplantats. Bei 6 Patienten mußte das Organ wegen irreversibler Abstoßung sofort entfernt werden. Bei den restlichen 19 Patienten mit primär erhaltenem Transplantat hat sich zur Blutstillung die Übernähung der Ruptur unter Verwendung körpereigener Muskelstückchen sehr bewährt. Die Verwendung von Fremdmaterial zur Sicherung der Naht erscheint überflüssig. Die Prognose der ehemals ruptierten Transplantation ist gut.

Schliisselwörter: Nierentransplantation - Nierentransplantatruptur. 